\title{
COMPUTATIONAL FLUID DYNAMICS FOR DENSE GAS-SOLID FLUIDIZED BEDS: A MULTI-SCALE MODELING STRATEGY
}

\author{
M. A. van der Hoef, M. van Sint Annaland and J. A. M. Kuipers* \\ Department of Science \& Technology, University of Twente, 7500 AE Enschede, The Netherlands \\ *Author to whom correspondence should be addressed. E-mail: j.a.m.kuipers@utwente.nl
}

\begin{abstract}
Dense gas-particle flows are encountered in a variety of industrially important processes for large scale production of fuels, fertilizers and base chemicals. The scale-up of these processes is often problematic and is related to the intrinsic complexities of these flows which are unfortunately not yet fully understood despite significant efforts made in both academic and industrial research laboratories. In dense gas-particle flows both (effective) fluid-particle and (dissipative) particle-particle interactions need to be accounted for because these phenomena to a large extent govern the prevailing flow phenomena, i.e. the formation and evolution of heterogeneous structures. These structures have significant impact on the quality of the gas-solid contact and as a direct consequence thereof strongly affect the performance of the process. Due to the inherent complexity of dense gas-particles flows, we have adopted a multi-scale modeling approach in which both fluid-particle and particle-particle interactions can be properly accounted for. The idea is essentially that fundamental models, taking into account the relevant details of fluid-particle (lattice Boltzmann model) and particle-particle (discrete particle model) interactions, are used to develop closure laws to feed continuum models which can be used to compute the flow structures on a much larger (industrial) scale. Our multi-scale approach (see Fig. 1) involves the lattice Boltzmann model, the discrete particle model, the continuum model based on the kinetic theory of granular flow, and the discrete bubble model. In this paper we give an overview of the multi-scale modeling strategy, accompanied by illustrative computational results for bubble formation. In addition, areas which need substantial further attention will be highlighted.
\end{abstract}

Keywords dense gas-solid flow, gas-fluidized beds, multi-scale modelling

\section{Introduction}

Dense gas-particle flows are frequently encountered in a variety of industrially important gas-solid contactors, of which the gas-fluidized bed can be mentioned as a very important example. Due to their favorable mass and heat transfer characteristics, gas-fluidized beds are often applied in the chemical, petrochemical, metallurgical, environmental and energy industries in large scale operations involving i.e. coating, granulation, drying, and synthesis of fuels and base chemicals (Kunii \& Levenspiel, 1991). Lack of understanding of the fundamentals of dense gas-particle flows, and in particular of the effects of gas-particle drag and particle-particle interactions (Kuipers et al., 1998; Kuipers \& van Swaaij, 1998), has led to severe difficulties in the scale-up of these industrially important gas-solid contactors (van Swaaij, 1990). To arrive at a better understanding of these complicated systems in which both gas-particle and particle-particle interactions play a dominant role, computer models have become an indispensable tool. However, the prime difficulty with modeling gas-fluidized beds is the large separation of scales: the largest flow structures can be of the order of meters; yet these structures are found to be directly influenced by details of the particle-particle collisions, which take place on the scale of millimeters or less. Therefore, we have adopted a multi-level modeling strategy (see Fig.1), with the prime goal to (i) obtain a fundamental insight in the complex dynamic behavior of dense gas-particle fluidized suspensions; that is, to gain an understanding based on elementary physical principles such as drag, friction and dissipation (ii) based on this insight, develop models with predictive capabilities for dense gas-particle flows encountered in engineering scale equipment. To this end, we consider gas-solid flows at four distinctive levels of modeling.

At the most detailed level of description the gas flow field is modeled at scales smaller than the size of the solid particles. The interaction of the gas phase with the solid phase is incorporated by imposing "stick" boundary conditions at the surface of the solid particles. This model thus allows us to measure the effective momentum exchange between the two phases, which can be used in the higher scale models. In our model, the flow field between spherical particles is solved by the lattice Boltzmann model (Succi, 2001; Ladd \& Verberg, 2001) although in principle other methods (such as standard computational fluid dynamics) could be used as well. At the intermediate level of description the flow field is modeled at a scale larger than the size of the particles, where a grid cell typically contains $\mathrm{O}\left(10^{2}\right) \sim \mathrm{O}\left(10^{3}\right)$ particles, which are assumed to be perfect spheres (diameter $d$ ). This model consists of two parts: a Lagrangian code for updating the positions and velocities of the solid particles from Newton's law, and a Eulerian code for updating the local gas density and velocity from the Navier-Stokes equation (Hoomans et al., 1996). The advantage of this Discrete Particle Model (DPM) is that it can account for particle-wall and particle-particle interactions in a realistic manner, for system sizes of about $O\left(10^{6}\right)$ particles, which is sufficiently large to allow for a direct 


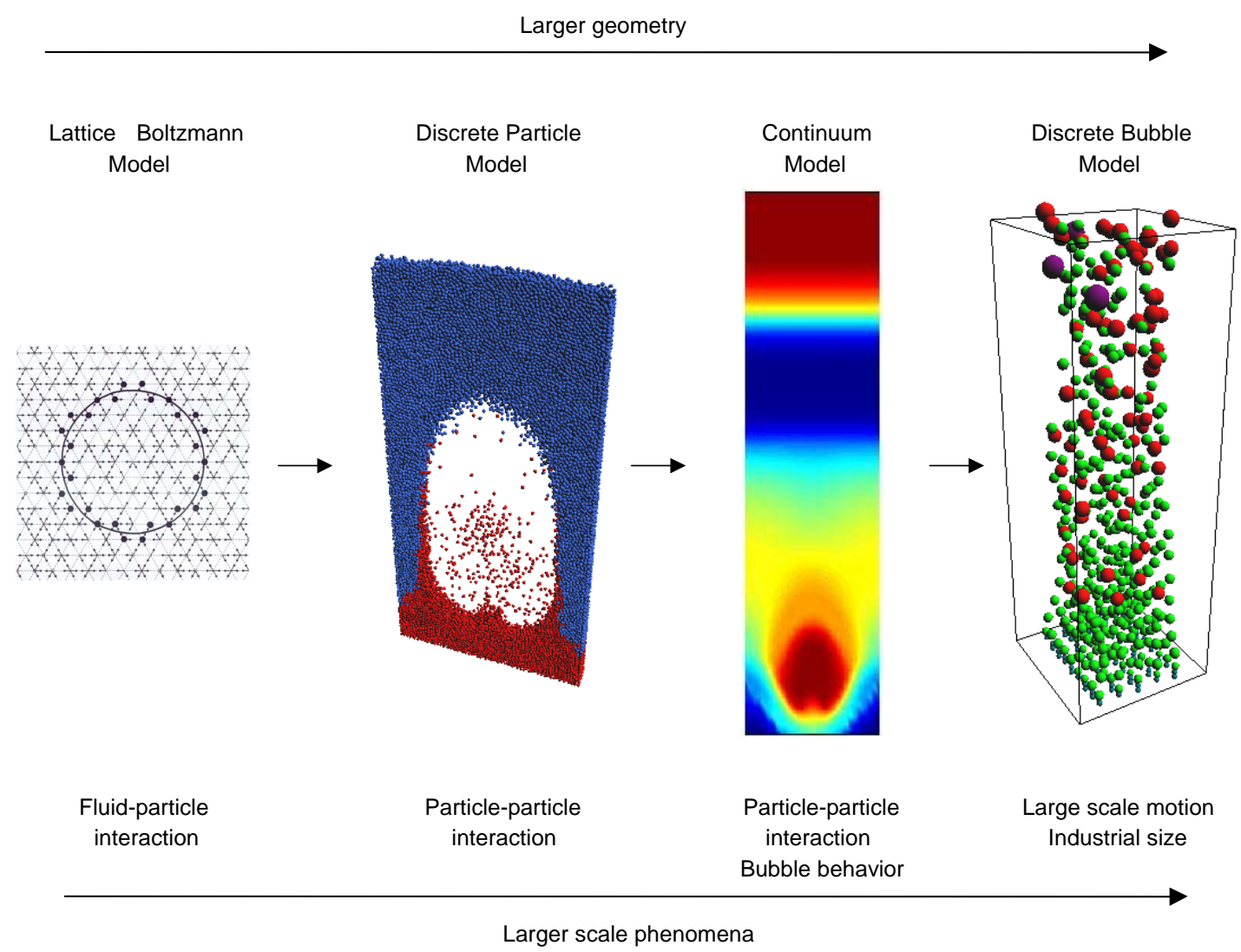

Fig. 1 Multi-level modeling scheme for dense gas-fluidized beds.

comparison with laboratory scale experiments. As a logical consequence of this approach a closure law for the effective momentum exchange has to be specified, which can be obtained from the aforementioned lattice Boltzmann simulations. Note that in chemical engineering, to date mainly empirical relations are used for the friction coefficient $\beta$ (defined by (1) and (2)), such as the Ergun (1952) correlation for porosities $\varepsilon<0.8$ :

$$
\frac{\beta d^{2}}{\mu}=150 \frac{(1-\varepsilon)^{2}}{\varepsilon}+1.75 \frac{(1-\varepsilon)}{\varepsilon} R e,
$$

and the Wen and $\mathrm{Yu}$ (1966) equation for porosities $\varepsilon>0.8$ :

$$
\begin{gathered}
\frac{\beta d^{2}}{\mu}=\frac{3}{4} C_{\mathrm{d}} \operatorname{Re}(1-\varepsilon) \varepsilon^{-2.65}, \\
C_{\mathrm{d}}=\left\{\begin{array}{ll}
24\left(1+0.15 R e^{0.687}\right) / \operatorname{Re} & \operatorname{Re}<10^{3} \\
0.44 & \operatorname{Re}>10^{3}
\end{array},\right.
\end{gathered}
$$

where $\mu$ is the viscosity of the gas phase, $R e$ is the particle Reynolds number, and $C_{\mathrm{d}}$ the drag coefficient, for which the expression of Schiller and Nauman (1935) is used. At an even larger scale a continuum description is employed for the solid phase, i.e. the solid phase is not described by individual particles, but by a local density and velocity field.
Hence, in this model both the gas-phase and the solid phase are treated on an equal footing, and for both phases an Eulerian code is used to describe the time evolution (see Kuipers et al., 1992; Gidaspow, 1994, amongst others). The information obtained in the two smaller-scale models is then included in the continuum models via the kinetic theory of granular flow. The advantage of this model is that it can predict the flow behavior of gas-solid flows at life-size scales, and these models are therefore widely used in commercial fluid flow simulators of industrial scale equipment. Finally, at the largest scale, the (larger) bubbles that are present in gas-solid fluidized beds are considered as discrete objects, similar to the solid particles in the DPM model. This model is an adapted version of the discrete bubble model for gas-liquid bubble columns. We want to stress that this model, as outlined in section 5 , has been developed quite recently, and the results should be considered as very preliminary. In this paper we will give an overview of these four levels of modeling as they are employed in our research group. In the following sections we will describe each of these models in more detail.

\section{Lattice Boltzmann Model (LBM)}

The lattice Boltzmann model (LBM) originates from the lattice-gas cellular automata (LGCA) models (Frisch et al., 
1986) for simple fluids. The LGCA model is basically a discrete, simplified version of the molecular dynamics model, which involves propagations and collisions of particles on a lattice. LGCA models have proved a simple and efficient way to simulate a simple fluid at the microscopic level, where it has been demonstrated both numerically and theoretically that the resulting macroscopic flow fields obey the Navier-Stokes equation. The lattice Boltzmann model is the ensemble averaged version of the LGCA model, so that it represents a propagation and collision of the particle distributions instead of the actual particles as in the LGCA models (McNamara \& Zanetti, 1988). From a macroscopic point of view, the LB model can be regarded as a finite difference scheme that solves the Boltzmann equation, the fundamental equation in the kinetic theory which underlies the equations of hydrodynamics. In its most simple form the finite difference scheme reads:

$$
f\left(\boldsymbol{v}, \boldsymbol{r}+\boldsymbol{c}_{i} \delta t, t+\delta t\right)-f\left(\boldsymbol{c}_{i}, \boldsymbol{r}, t\right)=\frac{\delta t}{\tau}\left(f\left(\boldsymbol{c}_{i}, \boldsymbol{r}, t\right)-f^{\mathrm{eq}}\left(\boldsymbol{c}_{i}, \boldsymbol{r}, t\right)\right),
$$

where $f$ is the single particle distribution function, which is equivalent to the fluid density in the 6 dimensional velocity-coordinate space, and $f^{\text {eq }}$ represents the equilibrium distribution. In Eq. (3), the position $\boldsymbol{r}$ and velocity $\boldsymbol{c}_{i}$ are discrete, i.e. the possible positions are restricted to the sites of a lattice, and thus the possible velocities are the vectors $c_{i}(i=1, b)$ connecting the $b$ nearest neighbor sites of this lattice. Note that Eq. (3) represents a propagation, followed by a "collision" (relaxation to the equilibrium distribution). From the single particle distribution function, the hydrodynamic variables of interest - the local gas density $\rho$ and velocity $\boldsymbol{c}_{i}$ - are obtained by summing up over all possible velocities:

$$
\rho(\boldsymbol{r}, t)=\sum_{i=1}^{b} f\left(\boldsymbol{c}_{i}, r, t\right), \quad \rho(\boldsymbol{r}, t) \boldsymbol{u}(\boldsymbol{r}, t)=\sum_{i=1}^{b} \boldsymbol{c}_{i} f\left(\boldsymbol{c}_{i}, \boldsymbol{r}, t\right) .
$$

It can be shown that the flow fields obtained from the LB model are - to order $\delta t^{2}$ - equivalent to those obtained from the Navier-Stokes equation, where the viscosity is set by the relaxation time $\tau$. One of the advantages of the LB model over other finite difference models for fluid flow, is that boundary conditions can be modeled in a very simple way. This makes the method particularly suit to simulate large moving particles suspended in a fluid phase. An obvious choice of the boundary condition is where the gas next to the solid particle moves with the local velocity of the surface of the solid particle, i.e. the so-called "stick" boundary condition. For a spherical particle suspended in an infinite three-dimensional system, moving with velocity $\boldsymbol{v}$, this condition will give rise to a frictional force on the particle $F=3 \pi \mu d \boldsymbol{v}$, at least in the limit of low particle Reynolds numbers $R e=\rho d \varepsilon v / \mu$, where $d$ is the hydrodynamic diameter of the particle, and $\mu$ is the shear viscosity. A particular efficient and simple way to enforce stick boundary conditions for static particles in the LB model is to let the distributions "bounce back" at the boundary nodes (Ladd \& Verberg, 2001); these nodes are defined as the points halfway the two lattice sites which are closest to the actual surface of the particle. The "bounce-back" rule means that the distribution function moves back into the direction that it comes from (see Fig. 2):

$$
f_{i}(r, t+1)=f_{i^{\prime}}(r, t) \text {, }
$$

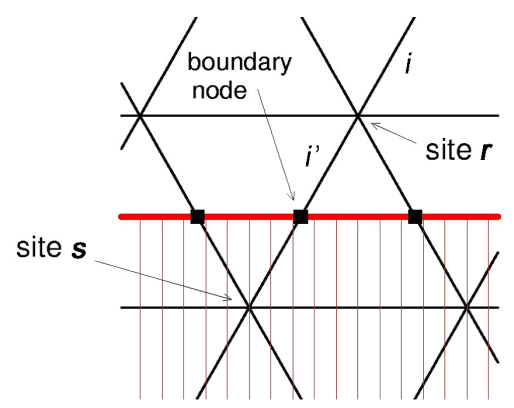

Fig.2 Illustration of the bounce-back rule. The distribution at site $r$ that moves at time $t$ into direction $i$, instead of arriving at the (virtual) site $\boldsymbol{s}$, is bounced at the boundary node, and thus arrives back at site $r$ at time $t+1$, but now headed in the opposite direction.

where $i$ and $i^{\prime}$ are opposite links. This rule ensures that the fluid velocity at the boundary node indeed vanishes: the momentum at the boundary node at time $t+1 / 2$ is given by:

$$
\rho_{b} u_{b}=f_{i^{\prime}}(r, t) c_{i^{\prime}}+f_{i}(r, t+1) c_{i},
$$

Inserting Eq. (5) and using $c_{i}=-c_{i}$, gives that $\rho_{b} u_{b}=0$. For non-static particles, the local fluid velocity must be set equal to the local boundary velocity $v_{b}$. This can be achieved by a simple modification of the bounce-back rule:

$$
f_{i}(r, t+1)=f_{i^{\prime}}(r, t)+\boldsymbol{a} \cdot \boldsymbol{c}_{i},
$$

where $a$ is chosen such that $\boldsymbol{u}_{b}=\boldsymbol{v}_{b}$. Note that only the component of $v_{b}$ in the direction of the link can be set in this way. For details we refer to Ladd and Verberg (2001). The drag force $\boldsymbol{F}_{\mathrm{d}}$ can also be directly measured in the simulation, from the change in gas momentum due to the boundary rules. In this way, the average drag force $\left\langle F_{\mathrm{d}}\right\rangle$ on a sphere in a static random array can be obtained, where the gas flow is set at a constant velocity $u_{0}$, according to the desired $R e$ number. The friction coefficient $\beta$ follows then from:

$$
\beta=\frac{1-\varepsilon}{V_{p}} \frac{\left\langle F_{d}\right\rangle}{u_{0}}, V_{p}=\frac{1}{6} \pi d^{3} .
$$

By using this method, we found for low Reynolds number excellent agreement with data obtained by multipole expansion methods (van der Hoef et al., 2005). By contrast, it was found that the widely used empirical correlations (1) and (2) significantly underestimate the drag force, at least for low Reynolds numbers. Based on the Carman-Kozeny approximation, we derived an expression for the correction of the monodisperse drag force to account for bidispersity which only depends on $y_{i}=d_{i} /\langle d\rangle$ with $\langle d\rangle$ the average 
diameter, for details see van der Hoef et al. (2005). In Fig. 3 we present some LBM results for a binary mixture at finite Reynolds numbers. In this figure the individual drag force $F_{\mathrm{i}}$ divided by the drag force $F(\phi)$ of a monodisperse system at the same solids volume fraction $\phi$, is plotted as a function of the correction factor $(1-\phi) y_{i}+\phi y_{i}^{2}$ that we derived, where $F(\phi)$ is our best fit to LBM simulation data for monodisperse systems:

$$
F(\phi)=10 \frac{\phi}{(1-\phi)^{2}}+(1-\phi)^{2}[1+1.5 \sqrt{\phi}] .
$$

As can be seen from Fig. 3, we find excellent agreement between our data and theory. It should be noted here that the assumption $F_{i}=F(\phi)$, which is currently used in literature can lead to differences with the LBM simulation data up to a factor of 5 . This finding indicates that one should be cautious with relying on ad hoc modifications of drag laws for monodisperse systems to extend their "validity" to polydisperse systems. In addition, this result highlights the usefulness of microscopic simulation methods, because the experimental determination of the individual effective drag force in a dense assembly would be extremely difficult.
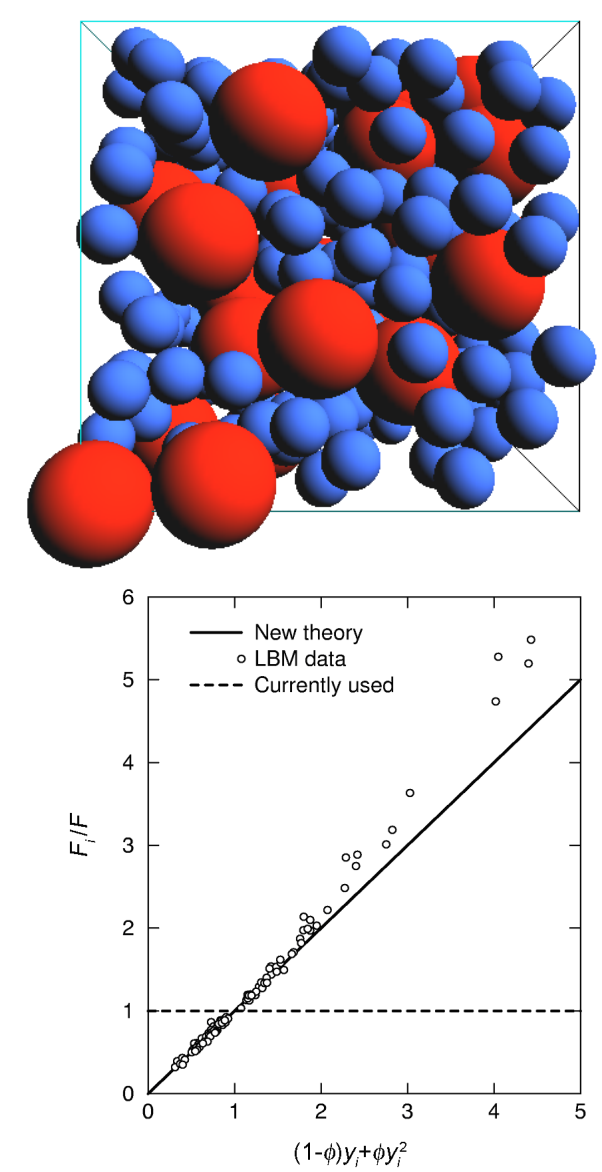

Fig. 3 Example of particle configuration generated with a Monte Carlo procedure for a binary system (upper) and dimensionless drag force computed for small and large particles from LBM (lower).

\section{Discrete Particle Model (DPM)}

The discrete particle model is one level higher in the multi-scale hierarchy. The most important difference with the lattice Boltzmann model is that in this model the size of the particles is smaller than the grid size that is used to solve the equations of motion of the gas phase. This means that for the interaction with the gas phase, the particles are simply point sources and sinks of momentum, where the finite volume of the particles only comes in via an average gas fraction in the drag force relations. A second (technical) difference with the LB model is that the evolution of the gas phase now follows from a finite difference scheme of the Navier-Stokes equation, rather than the Boltzmann equation. A complete description of the method can be found in Hoomans et al. (1996), however, we will briefly discuss some of the basic elements here. The discrete particle model consists of two parts: a Lagrangian part for updating the positions and velocities of the solid particles, and an Eulerian part for updating the local gas density and velocity. In the Lagrangian part, the equation of motion of each particle $i$ (velocity $\vec{v}_{i}$, mass $m_{i}$, volume $V_{i}$ ) is given by Newton's law

$$
m_{i} \frac{\mathrm{d} \vec{v}_{i}}{\mathrm{~d} t}=m_{i} \vec{g}+\frac{V_{i} \beta}{(1-\varepsilon)}\left(\vec{u}-\vec{v}_{i}\right)-V_{i} \nabla p+\vec{F}_{i}^{\mathrm{pp}}+\vec{F}_{i}^{\mathrm{pw}}
$$

where the RHS represents the total force acting on the particle. This includes external forces (the gravitational force $m_{i} \vec{g}$ ), interaction forces with the gas phase (drag force $\sim \beta\left(\vec{u}-\vec{v}_{i}\right)$ and pressure force $\left.V_{i} \nabla p\right)$, and finally the particle-particle forces $\vec{F}_{i}^{\text {pp }}$ and particle-wall forces $\vec{F}_{i}^{\text {pw }}$, which represents the momentum exchange during collisions, and possible long-range attractions between the particles, and particles and walls, respectively. There are, in principle, two ways to calculate the trajectories of the solid particles from Newton's law. In a time-driven numerical simulation, the new position $\vec{r}_{i}(t+\mathrm{d} t)$ and velocity $\vec{v}_{i}(t+\mathrm{d} t)$ are calculated from the values at time $t$, via a standard integration scheme for ODE's. Such type of simulation is in principle suitable for any type of interaction force between the particles. In an event-driven simulation, the interactions between the particles are considered instantaneous ("collisions"), and the systems evolves directly ("free flight") from nearest collision event to next-nearest collision event, etc. This method is efficient for low-density systems, however it is not suitable for dense-packed systems, or systems with long-range forces. In the Eulerian part of the code, the evolution of the gas phase is determined by the volume-averaged Navier-Stokes equations:

$$
\begin{gathered}
\frac{\partial}{\partial t}(\varepsilon \rho)+\nabla \cdot \varepsilon \rho \vec{u}=0, \\
\frac{\partial}{\partial t}(\varepsilon \rho \vec{u})+\nabla \cdot \varepsilon \rho \vec{u} \vec{u}=-\varepsilon \nabla p-\nabla \cdot \varepsilon \overline{\bar{\tau}}-\vec{S}+\varepsilon \rho \vec{g},
\end{gathered}
$$

where $\overline{\bar{\tau}}$ is the usual stress tensor, which includes the 
coefficient of shear viscosity. Note that there is a full two-way coupling with the Lagrangian part, i.e., the reaction from drag and pressure forces on the solid particles is included in the momentum equation for the gas phase via a source term $\vec{S}$ :

$$
\vec{S}=\frac{1}{V} \int \sum_{i} \frac{V_{i} \beta}{1-\varepsilon}\left(\vec{u}-\vec{v}_{i}\right) \delta\left(\vec{r}-\vec{r}_{i}\right) \mathrm{d} V .
$$

Equations (11) and (12) are solved with a semi-implicit method for pressure linked equations (SIMPLE-algorithm), with a time step that is in general an order of magnitude larger than the time step used to update the particle positions and velocities. The strength of the DP model is that it allows to study the effect of the particle-particle interactions on the fluidization behavior. In the most detailed model of description, the interparticle contact forces includes normal and tangential repulsive forces (modeled by linear springs), and dissipative forces (modeled by "dash pots"), and tangential friction forces (Walton, 1993). A DPM simulation study by Hoomans et al. (1996) showed that the heterogeneous flow structures in dense gas-fluidized beds are partly due to the collisional energy dissipation. More recently, Li and Kuipers (2003) demonstrated that such flow structures are also strongly influenced by the degree of non-linearity of the particle drag with respect to the gas fraction $\varepsilon$. Bokkers et al. (2004a; 2004b) studied the effect of the closures for gas-particle drag on the bubble-induced mixing in a pseudo 2D gas-fluidized bed and found that the best agreement between theory and experiment was obtained in case the LBM-generated drag closures reported by Hill et al. (2001a; 2001b) were used in their DPM simulations.

One of the great advantages of discrete particle simulations is that it allows to study properties of the system that are very diificult to obtain via experimentation. A particularly important example is the velocity distribution of the particles, i.e. the probability of finding a particle with a velocity component $v_{\alpha}$ with $\alpha=x, y, z$. It would be extremely difficult to obtain reliable estimates for the velocity distribution from experiments; yet, this function is of great relevance for the validity of the higher scale two-fluid model (see next section) derived from the kinetic theory, where it is assumed that the velocity distribution is both isotropic and nearly Gaussian. The discrete particle simulations are an ideal tool for testing this assumption, since it is relatively straightforward to measure the velocity distribution as all particle velocities are known at any moment in time.

We have studied the velocity distribution for two cases: in Fig. 4 we show the result for a fluidized bed of ideal (i.e. perfectly smooth and elastic) and non-ideal (i.e. rough and inelastic) particles. The system contained 25000 particles of $2.5 \mathrm{~mm}$ diameter, where the gas velocity is set to 1.5 times the minimum fluidization velocity. Details of the sampling procedure for obtaining the velocity distributions can be found in Goldschmidt et al. (2002). Figure 4 shows that for both ideal and non-ideal particles, the velocity distributions do not deviate significantly from a Gaussian and Maxwellian distribution. However, Fig. 4 reveals a clear anisotropy of the distribution in case of non-ideal particles. A possible explanation is the formation of dense particle clusters in the case of inelastic collisions, which may disturb the spatial homogeneity and thereby causing collisional anisotropy. Analysis (Jenkins \& Savage, 1983) of the normal and tangential component of the impact velocity indeed showed that, in dense gas-fluidized beds, not all impact angles occur with the same frequency.
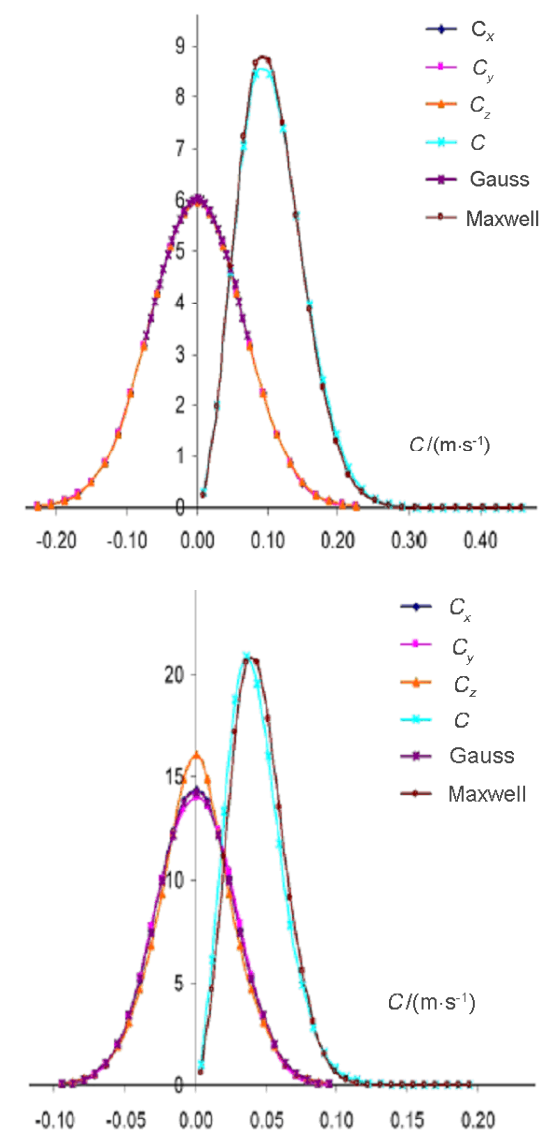

Fig. 4 DPM simulation data for the normalized particle velocity distribution $f_{x}\left(C_{x}\right), f_{y}\left(C_{y}\right), f_{z}\left(C_{z}\right)$ and $f(C)$, compared to a Gaussian/Maxwellian distribution. Upper graph: ideal particles; lower graph: non-ideal particles.

\section{Two-Fluid Model (TFM)}

The maximum number of particles that can be simulated with the DP model, as described in the previous section, is typically less than a million, whereas the number of particles that are present in an industrial size fluidized bed can be two to three orders of magnitude higher. Since both the CPU time and the required memory scales linear with the number of particles, it is obvious that DPM simulations of industrial size fluidized beds are beyond the capability of commercially available computer facilities within the foreseeable future. Therefore, a different type of model is used for simulations at larger scales, where the concept of a solid phase consisting of individual, distinguishable particles is abandoned. This so-called two-fluid continuum 
model (TFM) describes both the gas phase and the solids phase as fully inter-penetrating continua, using a set of generalized Navier-Stokes equations (Kuipers et al., 1992; Gidaspow, 1994). That is, the time evolution of the gas phase is still governed by (11) and (12); for the solid phase, the discrete particle part (10) is now replaced by a set of continuum equations of the same form as (11) and (12):

$$
\begin{gathered}
\frac{\partial}{\partial t}\left(\varepsilon_{\mathrm{s}} \rho_{\mathrm{s}}\right)+\nabla \cdot \varepsilon_{\mathrm{s}} \rho_{\mathrm{s}} \vec{v}=0 \\
\frac{\partial}{\partial t}\left(\varepsilon_{\mathrm{s}} \rho_{\mathrm{s}} \vec{v}\right)+\nabla \cdot \varepsilon_{\mathrm{s}} \rho_{\mathrm{s}} \vec{v} \vec{v}=-\varepsilon_{\mathrm{s}} \nabla p-\nabla p_{\mathrm{s}}-\nabla \cdot \varepsilon_{\mathrm{s}} \overline{\bar{\tau}}_{\mathrm{s}}+\vec{S}+\varepsilon_{\mathrm{s}} \rho_{\mathrm{s}} \vec{g}
\end{gathered}
$$

with $\rho_{\mathrm{s}}, \vec{v}$ and $\varepsilon_{\mathrm{s}}=1-\varepsilon$ the local density, velocity, and volume fraction of the solid phase, respectively. In this description the source term $\vec{S}$ is slightly different from (13), namely

$$
\vec{S}=\beta(\vec{u}-\vec{v}) .
$$

Obviously, the numerical scheme for updating the solid phase is now completely analogous to (and synchronous with) that of the gas phase. Since the concept of particles has disappeared completely in such a modeling, the effect of particle-particle interactions can only be included indirectly, via an effective solids pressure and effective solids viscosity. A description which allows for a slightly more detailed description of particle-particle interactions follows from the kinetic theory of granular flow (KTGF); such theory expresses the diagonal and off-diagonal elements of the solids stress tensor (i.e. the solids pressure and solids shear rate) as a function of the granular temperature for a monodisperse particle system, defined as:

$$
\theta=\frac{1}{3}\left\langle\vec{C}_{\mathrm{p}} \cdot \vec{C}_{\mathrm{p}}\right\rangle
$$

where $\vec{C}_{\mathrm{p}}$ represents the particle fluctuation velocity and the brackets indicate ensemble-averaging. The time evolution of the granular temperature itself is given by:

$$
\begin{aligned}
& \frac{3}{2}\left[\frac{\partial}{\partial t}\left(\varepsilon_{\mathrm{s}} \rho_{\mathrm{s}} \theta\right)+\nabla \cdot\left(\varepsilon_{\mathrm{s}} \rho_{\mathrm{s}} \theta \vec{v}\right)\right]= \\
& -\left(p_{\mathrm{s}} \overline{\bar{I}}+\varepsilon_{\mathrm{s}} \overline{\bar{\tau}}_{\mathrm{s}}\right): \nabla \vec{v}-\nabla \cdot\left(\varepsilon_{\mathrm{s}} \vec{q}_{\mathrm{s}}\right)-3 \beta \theta-\gamma,
\end{aligned}
$$

with $\vec{q}_{s}$ the kinetic energy flux, and $\gamma$ the dissipation of kinetic energy due to inelastic particle collisions. In equations (14)-(18) there are still a number of unknown quantities (pressure, stress tensor, energy flux), which must be expressed in terms of the basic hydrodynamic variables (density, velocity, granular temperature), in order to get a closed set of equations. The derivation of such constitutive equations follows from the KTGF, and can be found in the books by Chapman and Cowling (1970) and Gidaspow (1994) and the papers by Jenkins and Savage (1983) and Ding and Gidaspow (1990). In this work, the constitutive equations developed by Nieuwland et al. (1996) have been used for the particle phase rheology.

In Fig. 5 we show the simulated bubble formation for a pseudo two-dimensional (2D) bed (bed geometry: $0.57 \mathrm{~m} \times 0.015 \mathrm{~m} \times 1.0 \mathrm{~m}(w \times d \times h))$ operated with a central jet (diameter $0.015 \mathrm{~m}$ ) at a velocity of 40 times the incipient fluidization velocity. The bed contains ballotini with a particle diameter and density of $500 \mu \mathrm{m}$ and $\rho_{\mathrm{s}}=2660 \mathrm{~kg} \cdot \mathrm{m}^{-3}$ respectively. Clearly, a very complex bubble pattern results from the jet operation where the size and the shape of the formed bubbles continuously change. It can also clearly be seen that bubble coalescence occurs leading to a rapid increase in the bubble size.

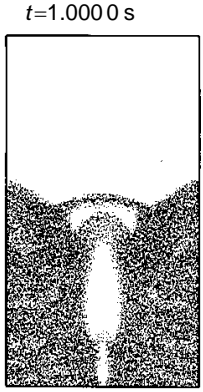

$t=6.0000 \mathrm{~s}$

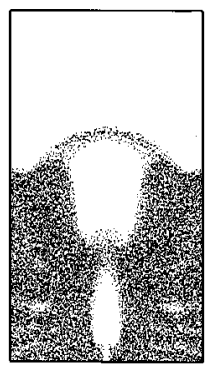

$t=2.0000 \mathrm{~s}$

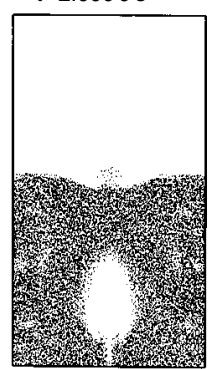

$t=7.0000 \mathrm{~s}$

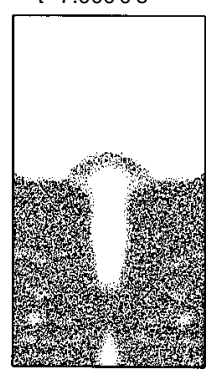

$t=3.0000 \mathrm{~s}$

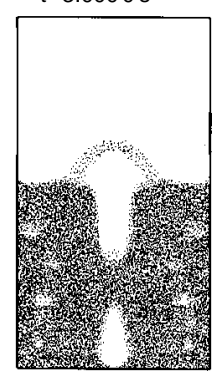

$t=8.0000 \mathrm{~s}$

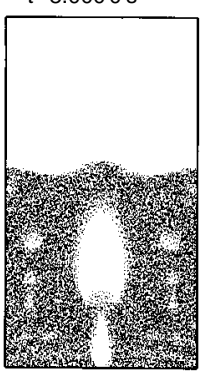

$t=4.0000 \mathrm{~s}$

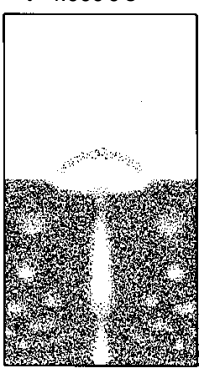

$t=9.0000 \mathrm{~s}$

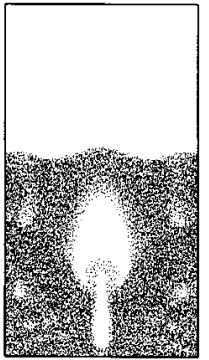

$t=5.0000 \mathrm{~s}$

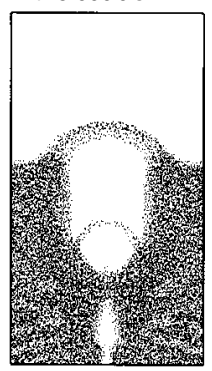

$t=10.0000 \mathrm{~s}$

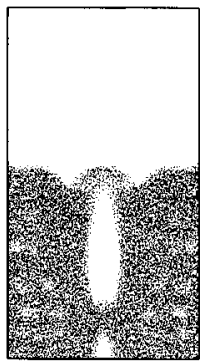

Fig. 5 Computed bubble formation in a pseudo 2D gas-fluidized bed with a central jet. Bed material: ballotini with $d_{\mathrm{p}}=500 \mu \mathrm{m}$ and $\rho_{\mathrm{s}}=2660 \mathrm{~kg} \cdot \mathrm{m}^{-3}$. Jet velocity: $10.0 \mathrm{~m} \cdot \mathrm{s}^{-1}\left(40 \mathrm{u}_{\mathrm{mf}}\right)$. 


\section{Discrete Bubble Model (DBM)}

Although the two fluid model can simulate fluidized beds at life-size scales, the largest scale industrial fluidized bed reactors (diameter 5 meters, height 16 meters) are still beyond its capabilities. However, it is possible to introduce yet another upscaling by considering the bubbles as discrete entities, as observed in the DPM and TFM models of gas-fluidized beds. This is the so-called discrete bubble model, which has been successfully applied in the field of gas-liquid bubble columns (Delnoij et al., 1997). The idea to apply this model to describe the large scale solids circulation that prevails in gas-solid reactors is new, however. In this paper, we want to show some first results of the discrete bubble model applied to gas-solid systems, which involves some slight modifications of the equivalent model for gas-liquid systems. To this end the emulsion phase is modeled as a continuum, like the liquid in a gas-liquid bubble column, and the larger bubbles are treated as discrete bubbles. Note that granular systems have no surface tension, so in that respect there is a pronounced difference with the bubbles present in gas-liquid bubble columns. For instance, the gas will be free to flow through a bubble in the gas-solid systems, which is not the case for gas-liquid systems. As far as the numerical part is concerned, the DBM strongly resembles the discrete particle model as outlined in section 3 , since it is also of the Euler-Lagrange type with the emulsion phase described by the volume-averaged Navier-Stokes equations:

$$
\begin{gathered}
\frac{\partial}{\partial t}(\varepsilon \rho)+\nabla \cdot \varepsilon \rho \vec{u}=0, \\
\frac{\partial}{\partial t}(\varepsilon \rho \vec{u})+\nabla \cdot \varepsilon \rho \vec{u} \vec{u}=-\varepsilon \nabla p-\nabla \cdot \varepsilon \overline{\bar{\tau}}-\vec{S}+\varepsilon \rho \vec{g},
\end{gathered}
$$

whereas the discrete bubbles are tracked individually according to Newton's second law of motion:

$$
m_{\mathrm{b}} \frac{\mathrm{d} \vec{v}_{\mathrm{b}}}{\mathrm{d} t}=\vec{F}_{\text {tot }}
$$

where $\vec{F}_{\text {tot }}$ is the sum of different forces acting on a single bubble:

$$
\vec{F}_{\text {tot }}=\vec{F}_{\mathrm{g}}+\vec{F}_{\mathrm{d}}+\vec{F}_{\mathrm{p}}+\vec{F}_{\mathrm{L}}+\vec{F}_{\mathrm{VM}}
$$

As in the DPM model, the total force on the bubble has contributions from gravity $\left(\vec{F}_{\mathrm{g}}\right)$, pressure gradients $\left(\vec{F}_{\mathrm{p}}\right)$ and drag from the interaction with emulsion phase $\left(\vec{F}_{\mathrm{d}}\right)$. For the drag force on a single bubble (diameter $d_{b}$ ), the correlations for the drag force on a single sphere are used, only with a modified drag coefficient $C_{d}$, such that it yields the Davies-Taylor relation $v_{b r}=0.711 \sqrt{g d_{b}}$ for the rise velocity of a single bubble. Note that in (21), there are two forces present which are not found in the DPM, namely the lift force $\vec{F}_{\mathrm{L}}$ and the virtual mass force $\vec{F}_{\mathrm{VM}}$. The lift force is neglected in this application, whereas the virtual mass force coefficient is set to 0.5 . An advantage of this approach to model large scale fluidized bed reactors is that the behaviour of bubbles in fluidized beds can be readily incorporated in the force balance of the bubbles. In this respect, one can think of the rise velocity, and the tendency of rising bubbles to be drawn towards the center of the bed, from the mutual interaction of bubbles and from wall effects (Kobayashi et al., 2000). Coalescence, which is an highly prevalent phenomenon in fluidized beds, can also be easily included in the DBM, since all the bubbles are tracked individually.

With the DBM, two preliminary calculations have been performed for industrial scale gas-phase polymerization reactors, in which we want to demonstrate the effect of the superficial gas velocities, set to $0.1 \mathrm{~m} \cdot \mathrm{s}^{-1}$ and $0.3 \mathrm{~m} \cdot \mathrm{s}^{-1}$. The geometry of the fluidized bed was $1.0 \mathrm{~m} \times 3.0 \mathrm{~m} \times 1.0 \mathrm{~m}$ $(w \times h \times d)$. The emulsion phase has a density of $400 \mathrm{~kg} \cdot \mathrm{m}^{-3}$ and the apparent viscosity was set to $1.0 \mathrm{~Pa} \cdot \mathrm{s}$. The density of the bubble phase was $25 \mathrm{~kg} \cdot \mathrm{m}^{-3}$. The bubbles were injected via 49 nozzles positioned equally distributed in a square in the middle of the column.

In Fig. 6 snapshots are shown of the bubbles that rise in the fluidized bed with a superficial velocity of $0.1 \mathrm{~m} \cdot \mathrm{s}^{-1}$ and $0.3 \mathrm{~m} \cdot \mathrm{s}^{-1}$, respectively. It is clearly shown that the bubble hold-up is much larger with a superficial velocity of $0.3 \mathrm{~m} \cdot \mathrm{s}^{-1}$. However, the number of bubbles in this case might be too large, since coalescence has not been taken into account in these simulations. In Fig. 6 in addition timeaveraged plots are shown of the emulsion velocity after
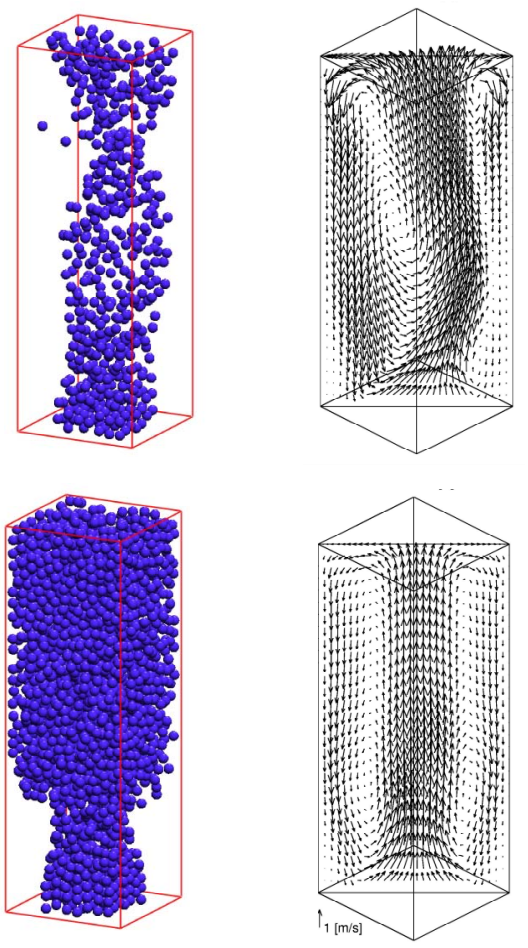

Fig. 6 Snapshots of the bubble configurations (left) computed from the DBM model without coalescence, and the time average vector plots of the emulsion phase (right) after $100 \mathrm{~s}$ of simulation; top: $u_{0}=0.1 \mathrm{~m} \cdot \mathrm{s}^{-1}, d_{\mathrm{b}}=0.04 \mathrm{~m}$; bottom: $u_{0}=0.3 \mathrm{~m} \cdot \mathrm{s}^{-1}, d_{\mathrm{b}}=0.04 \mathrm{~m}$. 
$100 \mathrm{~s}$ of simulation. The large convection patterns, upfow in the middle, and downflow along the wall, and the effect of the superficial gas velocity, are clearly demonstrated. Future work will be focused on implementation of closure equations in the force balance, like empirical relations for bubble rise velocities and the interaction between bubbles. The model can be augmented with energy balances to study temperature profiles in combination with the large circulation patterns.

\section{Summary and Outlook}

In this paper we have presented an overview of the multi-scale methods that we use to study gas-solid fluidized beds. The key idea is that the methods at the smaller, more detailed scale can provide qualitative and quantitative information which can be used in the higher scale models. A typical example of such qualitative information is the insight (from the DPM simulations) that inelastic collisions and nonlinear drag can lead to heterogeneous flow structures. Even more important, however, is the quantitative information that the smaller scale models can provide. A typical example of this is the drag force relation obtained from the LBM simulations, which finds its direct use in both the DPM and TFM simulations. We should note here that although the new drag force relations seem to give results at the DPM/TFM level which compare better with the experimental findings, these relations are still far from optimal. In particular, it should be borne in mind that these drag force relations are derived for static, unbounded, homogeneous arrays of mono-disperse spheres. Yet, at the DPM/TFM level these relations are applied to systems which are, even locally, inhomogeneous and non-static; furthermore, rather ad hoc modifications are used to allow for polydispersity. In future work, we want to focus on developing drag force relations for systems which deviate from the ideal conditions, where the parameters which would quantify such deviation may be trivial to define (polydispersity: width of the size distribution; moving particles: granular temperature) or not so trivial (inhomogeneities). Our lattice Boltzmann results for the drag force in binary systems (van der Hoef et al., 2005) revealed significant deviations with the ad hoc modifications of the monodisperse drag force relations, in which it is assumed that the drag force scales linearly with the particle diameter. At present, only qualitative information from the DPM simulations is obtained, such as the aforementioned heterogeneous flow structures, which is caused by dissipative forces.

Another example is the functional form of the velocity distribution. It was found that that dissipative interaction forces cause an anisotropy in the distribution, although the functional form remains close to Gaussian for all three directions (Goldschmidt et al., 2002). It would be interesting to include the effect of anisotropy at the level of the TFM, for instance along the lines of the kinetic theory developed by Jenkins and Richman (1988) for shearing granular flows. Although the continuum models have been studied extensively in the literature (e.g. Kuipers et al., 1992; Gidaspow, 1994), these models still lack the capability of describing quantitatively particle mixing and segregation rates in multi-disperse fluidized beds. An important improvement in the modeling of life-size fluidized beds could be made if direct quantitative information from the discrete particle simulations could find its way in the continuum models. In particular, it would be of great interest to find improved expressions for the solid pressure and the solid viscosity, as they are used in the two fluid model, however, it is a non-trivial task to extract direct data on the solid viscosity and pressure in a DPM simulation. A very simple, indirect method for obtaining the viscosity is to monitor the decay of the velocity of a large spherical intruder in the fluidized bed. The viscosity of the bed follows then directly from the Stokes-Einstein formula for the drag force. Very preliminary results - obtained from data of a high velocity impact — were in reasonable agreement with the experimental values for the viscosity. More elaborate simulations of these systems are currently underway.

Finally, the discrete bubble model applied to gas-solid systems seems to be a promising new approach for describing the large scale motion in life-size chemical reactors. Essential for this model to be successful is that reliable information with regard to rise velocities and mutual interaction of the bubbles is incorporated, which can be obtained from the lower scale simulations. In particular, the TFM and DPM simulations will be used to guide the formulation of additional rules to properly describe the coalescence of bubbles, which is at present not incorporated in the model. This will be the subject of future research.

\section{Acknowledgment}

The lattice Boltzmann simulations have been performed with the SUSP3D code developed by Anthony Ladd. We would like to thank him for making his code available, and Albert Bokkers for performing the DPM, TFM and DBM simulations.

\section{References}

Bokkers, G. A., van Sint Annaland, M. \& Kuipers, J. A. M. (2004a). Mixing and segregation in a bi-dispersed gas-solid fluidized bed: a numerical and experimental study. Powder Technol., 140, 176-186.

Bokkers, G. A., van Sint Annaland, M. \& Kuipers, J. A. M. (2004b). Comparison of continuum models using the kinetic theory of granular flow with discrete particle models and experiments: extent of particle mixing induced by bubbles. Proceedings of Fluidization XI (pp.187-194), May 9-14, 2004, Naples, Italy.

Chapman, S. \& Cowling, T. G. (1970). The Mathematical Theory of Non-uniform Gases (Trial mode edition). Cambridge: Cambridge University Press.

Delnoij, E., Kuipers, J. A. M. \& van Swaaij, W. P. M. (1997). Computational fluid dynamics applied to gas-liquid contactors. Chem. Eng. Sci., 52, 3623. PhD thesis, University of Twente, Enschede, The Netherlands.

Ding, J. \& Gidaspow, D. (1990). A bubbling fluidization model 
using kinetic theory of granular flow. AlChE J., 36, 523.

Ergun, S. (1952). Fluid flow through packed columns. Chem. Eng. Process., 48, 89.

Frisch, U., Hasslacher, B. \& Pomeau, Y. (1986). Lattice gas automata for the Navier-Stokes equation. Phys. Rev. Lett., 56, 1505.

Gidaspow, D. (1994). Multiphase Flow and Fluidization: Continuum and Kinetic Theory Descriptions. Boston: Academic Press.

Goldschmidt, M. J. V., Beetstra, R. \& Kuipers, J. A. M. (2002). Hydrodynamic modelling of dense gas-fluidised beds: comparison of the kinetic theory of granular flow with 3-D hardsphere discrete particle simulations. Chem. Eng. Sci., 57, 2059.

Hill, R. J., Koch, D. L. \& Ladd, A. J. C. (2001a). The first effects of fluid inertia on flow in ordered and random arrays of spheres. $J$. Fluid Mech., 448, 213.

Hill, R. J., Koch, D. L. \& Ladd, A. J. C. (2001b). Moderate-Reynolds-number flows in ordered and random arrays of spheres. $J$. Fluid Mech., 448, 243.

Hoomans, B. P. B., Kuipers, J. A. M., Briels, W. J. \& van Swaaij, W. P. M. (1996). Discrete particle simulation of bubble and slug formation in a two-dimensional gas-fluidized bed: a hard sphere approach. Chem. Eng. Sci., 51, 99.

Jenkins, J. T. \& Savage, S. B. (1983). A theory for the rapid flow of identical, smooth, nearly elastic particles. J. Fluid Mech., 130 187.

Jenkins, J. T. \& Richman, M. W. (1988). Plane simple shear flow of smooth inelastic circular disks: the anisotropy of the second moment in the dilute and dense limits. J. Fluid Mech., 192, 313.

Kobayashi, N., Yamazaki, R. \& Mori, S. (2000). A study on the behavior of bubbles and soldis in bubbling fluidized beds. Powder Technol., 113, 327.

Kuipers, J. A. M., van Duin, K. J., van Beckum, F. P. H. \& van Swaaij, W. P. M. (1992). A numerical model of gas-fluidized beds. Chem. Eng. Sci., 47, 1913.

Kuipers, J. A. M., Hoomans, B. P. B. \& van Swaaij, W. P. M (1998). Hydrodynamic modeling of gas-fluidized beds and their role for design and operation of fluidized bed chemical reactors. Proceedings of the Fluidization IX conference (pp.15-30), Durango, USA.

Kuipers, J. A. M. \& van Swaaij, W. P. M. (1998). Computational fluid dynamics applied to chemical reaction engineering. Adv. Chem. Eng., 24, 227.

Kunii, D. \& Levenspiel, O. (1991). Fluidization Engineering. Butterworth Heinemann series in Chemical Engineering, London.

Ladd, A. J. C. \& Verberg, R. (2001). Lattice-Boltzmann simulations of particle fluid suspensions. J. Stat. Phys., 104, 1191.

$\mathrm{Li}$, J. \& Kuipers, J. A. M. (2003). Gas-particle interactions in dense gas-fluidized beds. Chem. Eng. Sci., 58, 711.

McNamara, G. R. \& Zanetti, G. (1988). Use of the Boltzmann equation to simulate lattice-gas automata. Phys. Rev. Lett., 61, 2332.

Nieuwland, J. J., van Sint Annaland, M., Kuipers, J. A. M. \& van Swaaij, W. P. M. (1996). Hydrodynamic modeling of gas/particle flow in riser reactors. AlChE J., 42, 1569.

Schiller, L. \& Nauman, A. (1935). A drag coefficient correlation. V.D.I. Zeitung, 77, 318.

Succi, S. (2001). The Lattice Boltzmann Equation for Fluid dynamics and Beyond. Oxford: Oxford Science Publications.

van der Hoef, M. A., Beetstra, R. \& Kuipers, J. A. M. (2005). Lattice Boltzmann simulations of low Reynolds number flow past mono- and bidisperse arrays of spheres: results for the permeability and drag force. J. Fluid Mech., 528, 233-253.

van Swaaij, W. P. M. (1990). Chemical reactors. In Davidson, J. F. \& Clift, R. (Eds.), Fluidization. London: Academic Press.

Walton, O. R. (1993). Numerical simulation of inelastic, frictional particle-particle interactions. In Roco, M. C. (Ed.), Particulate Two-Phase Flow, Butterworth Heinemann series in Chemical Engineering, London.

Wen, C. Y. \& Yu, Y. H. (1966). Mechanics of fluidization. AlChE Symp. Ser., 62, 100.

Manuscript received January 10, 2005 and accepted April 1, 2005. 